\title{
Photocurrent pattern formation in polymer/methanofullerene blends imaged by near-field scanning photocurrent microscopy
}

\author{
C. R. McNeill ${ }^{\text {a) }}$ and P. C. Dastoor \\ School of Mathematical and Physical Sciences, The University of Newcastle, University Drive, Callaghan, \\ New South Wales 2308, Australia
}

(Received 28 June 2005; accepted 14 December 2005; published online 1 February 2006)

\begin{abstract}
The dimensional dependence of micron-sized current inhomogeneities in poly[2-methoxy-5(2'-ehtylhexyloxy)-1,4-phenylene vinylene]/1-(3-methoxycarbonyl)-propyl-1-1-phenyl-(6,6) $\mathrm{C}_{61}$ (MEH-PPV/PCBM) solar cells on processing conditions has been investigated. These current inhomogeneities typically consist of a central PCBM aggregate surrounded by a larger region of poor photocurrent production. For films spin coated from chlorobenzene, both the diameter of the current inhomogeneities and the size of the corresponding PCBM aggregates systematically increase with the time allowed for the film to dry. These observations indicate that the current inhomogeneities are a consequence of the nucleation and growth of the central PCBM cluster at the expense of PCBM from the surrounding area. In contrast to the results observed with chlorobenzene as the solvent, no micron-sized current inhomogeneities are observed using toluene as the solvent, despite the increased density of PCBM clusters in the film. The solubility of PCBM and the solvent volatility are identified as key parameters determining the formation of micron-sized current patterns. Also discussed is the influence of these current features on overall device performance, along with the influence of the film nanomorphology as processing conditions are changed. (C) 2006 American Institute of Physics. [DOI: 10.1063/1.2165414]
\end{abstract}

\section{INTRODUCTION}

The efficiency of polymer solar cells based on blends of conjugated polymer and fullerene has increased over the past decade with solar power conversion efficiencies of over $3 \%$ now reported. ${ }^{1-6}$ In the development of efficient polymerbased solar cells much has been made on the role of film morphology on device performance. In particular, following the discovery that spin coating blend films of poly[2methoxy-5-(3,7'-dimethloctyloxy)-1,4-phenylene vinylene] (MDMO-PPV) and 1-(3-methoxycarbonyl)-propyl-1-1phenyl- $(6,6) \mathrm{C}_{61}$ (PCBM) from chlorobenzene instead of toluene increases the efficiency by nearly threefold, ${ }^{4}$ the role of the so-called nanomorphology in determining overall device performance has been extensively investigated. ${ }^{7-10}$ In such reports, the efficiency of devices is typically compared to transmission electron microscope (TEM) and atomic force microscope (AFM) images of similarly prepared composite films that probe structural features on the length scale of less than $1 \mu \mathrm{m}$. However, recent studies using techniques that image device photocurrent have shown that there are variations in quantum efficiency on the length scale of tens to hundreds of micrometers. ${ }^{11,12}$ Jeranko et al., using a diffraction-limited laser scanning technique, have shown recently that even in high-efficiency devices there exist photocurrent patterns on the length scale of tens to hundreds of micrometers. ${ }^{11}$ In particular, devices yielding solar efficiencies of $2.6 \%$ were found to exhibit characteristic spots of $50-100 \mu \mathrm{m}$ in diameter where the photocurrent reaches only

\footnotetext{
a) Author to whom correspondence should be addressed; present address: Cavendish Laboratory, Madingley Road, Cambridge CB3 OHE, United Kingdom; electronic mail: crm51@cam.ac.uk
}

$50 \%$ of the current of surrounding areas. Additionally, photocurrent patterns were found to form as the devices aged. Recently, we have developed the near-field scanning photocurrent microscopy (NSPM) technique to probe photocurrent in polymer devices with subwavelength resolution. ${ }^{12}$ In addition to mapping the photocurrent with a resolution of 100 $\mathrm{nm},{ }^{13}$ the film morphology is simultaneously mapped thus bridging the measurements of Jeranko et al. that primarily measure photocurrent, and those using techniques such as AFM or TEM that measure film composition only. While we have been able to detect variations in photocurrent on the length scale of hundreds of nanometers, ${ }^{13}$ directly correlating the film nanomorphology with quantum efficiency, we have also measured variations in photocurrent on the length scale of tens of micrometers ${ }^{12}$ similar to Jaernko et al. In our previous communication, ${ }^{12}$ the observed micron-sized currentdepleted regions were invariably centered on protrusions from the film attributed to PCBM-rich aggregates. In order to further study the origin of these micron-sized low-efficiency spots we have studied the dependence of their size and distribution upon processing conditions. We show that these photocurrent spots increase in size with increasing evaporation time, and observe that the size of the central PCBM-rich peak also increases systematically with increasing depletion size. We therefore attribute these patterns to the nucleation and growth of PCBM-rich aggregates that form during spin coating, sourcing PCBM from the surrounding regions as the solution evaporates.

\section{EXPERIMENT}

In this experiment we used poly[2-methoxy-5(2'-ehtylhexyloxy)-1,4-phenylene vinylene] (MEH-PPV) 


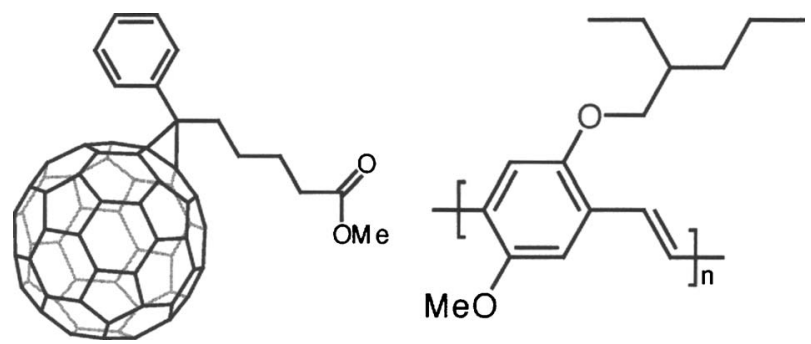

FIG. 1. Molecular structures of 1-(3-methoxycarbonyl)-propyl-1-1phenyl-(6,6) $\mathrm{C}_{61} \quad$ (PCBM) (left) and poly[2-methoxy-5(2'-ehtylhexyloxy)-1,4-phenylene vinylene (MEH-PPV) (right).

(see Fig. 1 for chemical structure) sourced from American Dye Source (Baie D'Urfe, Quebec, Canada) and was purified using the following procedure. Firstly, the polymer was dissolved in chlorobenzene by stirring overnight at $80^{\circ} \mathrm{C}$. After the solution was allowed to cool it was then added dropwise to an excess solution of methanol with stirring. The highweight precipitate that resulted was removed from the lowweight impurities that remained in solution by filtration ( $2 \mu \mathrm{m}$ pore size, Millipore). The reclaimed polymer was dried overnight under vacuum before redissolving. PCBM (Fig. 1) was sourced from Hummelen and co-workers at the University of Groningen and used as received. MEH-PPV/ PCBM blend solutions with a weight ratio of 1:4 (MEHPPV:PCBM) were prepared by dissolving the appropriate amounts of each material in either chlorobenzene or toluene and stirring overnight at $80^{\circ} \mathrm{C}$. A polymer concentration of $5 \mathrm{~g} / \mathrm{l}$ was used for these stock solutions.

Photovoltaic devices were prepared by spin coating poly(3,4-ethylenedioxythiophene):polystyrene sulfonic acid (PEDOT:PSS) ("BAYTRON P," H.C. Starck GmbH) onto cleaned indium tin oxide (ITO) glass slides (Delta Technologies Ltd., Stillwater, MN) with drying at $120{ }^{\circ} \mathrm{C}$ under flowing nitrogen. The roughness of the ITO substrates was not more than $10 \mathrm{~nm}$ as measured by AFM. The active layer was then deposited by spin coating the polymer/fullerene solution. The substrates were then transferred to a vacuum system where semitransparent films of silver were deposited at a pressure of less than $10^{-6}$ mbar. The thicknesses of the layers in all the devices tested were $140 \mathrm{~nm}$ (ITO), $85 \mathrm{~nm}$ (PEDOT:PSS), $\quad 70 \pm 15 \mathrm{~nm}$ (MEH-PPV/PCBM), and $30 \pm 5 \mathrm{~nm}$ (silver) as determined by profilometery (Tencor Alpha-Step 500). Films prepared from chlorobenzene were prepared with varying processing conditions as follows. A "cold-spun" film that was afforded a relatively long time to dry was prepared by spin coating from a solution at $4{ }^{\circ} \mathrm{C}$ at $1000 \mathrm{rpm}$. A "room-temperature-spun" film that was afforded a moderate amount of time to dry was prepared by spin coating from a solution at $25^{\circ} \mathrm{C}$ at $3000 \mathrm{rpm}$. Finally, a "hotspun" film that was allowed only a relatively short time to dry was prepared by spin coating from a solution at $80{ }^{\circ} \mathrm{C}$ at $5000 \mathrm{rpm}$ onto a heated substrate. All chlorobenzene solutions were taken from the same stock solution (see above), and the room-temperature and cold solutions were slightly diluted (by not more than 20\%) to ensure similar active layer thickness. Toluene films were spin coated from a solution at $80{ }^{\circ} \mathrm{C}$ at $3000 \mathrm{rpm}$.

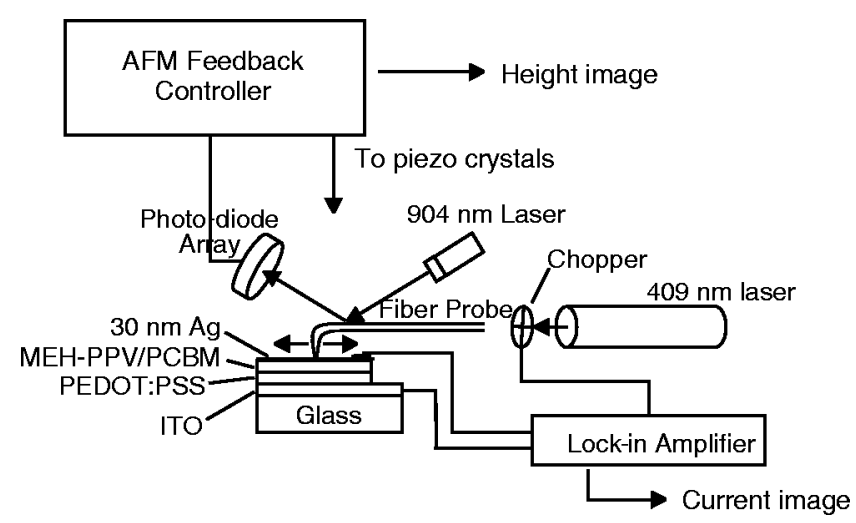

FIG. 2. Schematic diagram of the NSPM experimental setup for mapping the short-circuit current of polymer solar cells.

Details of the NSPM technique have been described elsewhere in detail. ${ }^{12}$ Briefly, a Nanonics SPM 100 near-field scanning optical microscope (NSOM) was used to scan a fiber probe across the surface of the semitransparent silver electrode using conventional AFM laser deflection feedback mode to generate height images (see Fig. 2). Photocurrent images were acquired simultaneously by measuring with a lock-in amplifier (Stanford Research Systems SR 830) the current produced by the device by excitation from chopped $409 \mathrm{~nm}$ laser light emitted from the end of the fiber probe. A $904 \mathrm{~nm}$ laser was used for AFM feedback detection to prevent unwanted excitation in the materials. No evidence for photoinduced degradation of the materials during scanning was observed. All devices for NSPM characterization were prepared in the same production run, and all measured on the same day. In particular, the same NSOM tip was used for all measurements with an aperture of $200 \mathrm{~nm}$ diameter providing a nominal lateral resolution of $200 \mathrm{~nm}$. Samples were stored in a nitrogen glove box prior to testing, and to avoid the remote possibility of aging effects skewing the results, the cold-spun device was measured first, followed by the room-temperature-spun device, the hot-spun device, and finally the toluence device.

Far-field photovoltaic characterization was performed on similarly prepared devices but with a calcium $(40 \mathrm{~nm}) /$ silver $(60 \mathrm{~nm})$ electrode structure. Illumination was provided by a Solux halogen lamp with an intensity of $80 \mathrm{~mW} / \mathrm{cm}^{2}$ measured at the device holder using a calibrated silicon photodiode. Electrical measurements were acquired using a Keithley 2400 source measure unit. Measurements were performed in air on devices encapsulated in a nitrogen glove box. For both near- and far-field measurements the active area of the device (determined by overlap of the electrodes) was $4 \mathrm{~mm}^{2}$.

\section{RESULTS AND DISCUSSION}

Figure 3 shows representative AFM and NSPM images of the four device types prepared, namely, the cold-spun device, the room-temperature-spun device, the hot-spun device, and the toluene device. For each device, five scans were taken at random locations on the device surface. The NSPM maps reveal that micron-sized current features are observed in all chlorobenzene films. Consistent with previous work, ${ }^{12}$ all of the large dark features in the NSPM images are cen- 

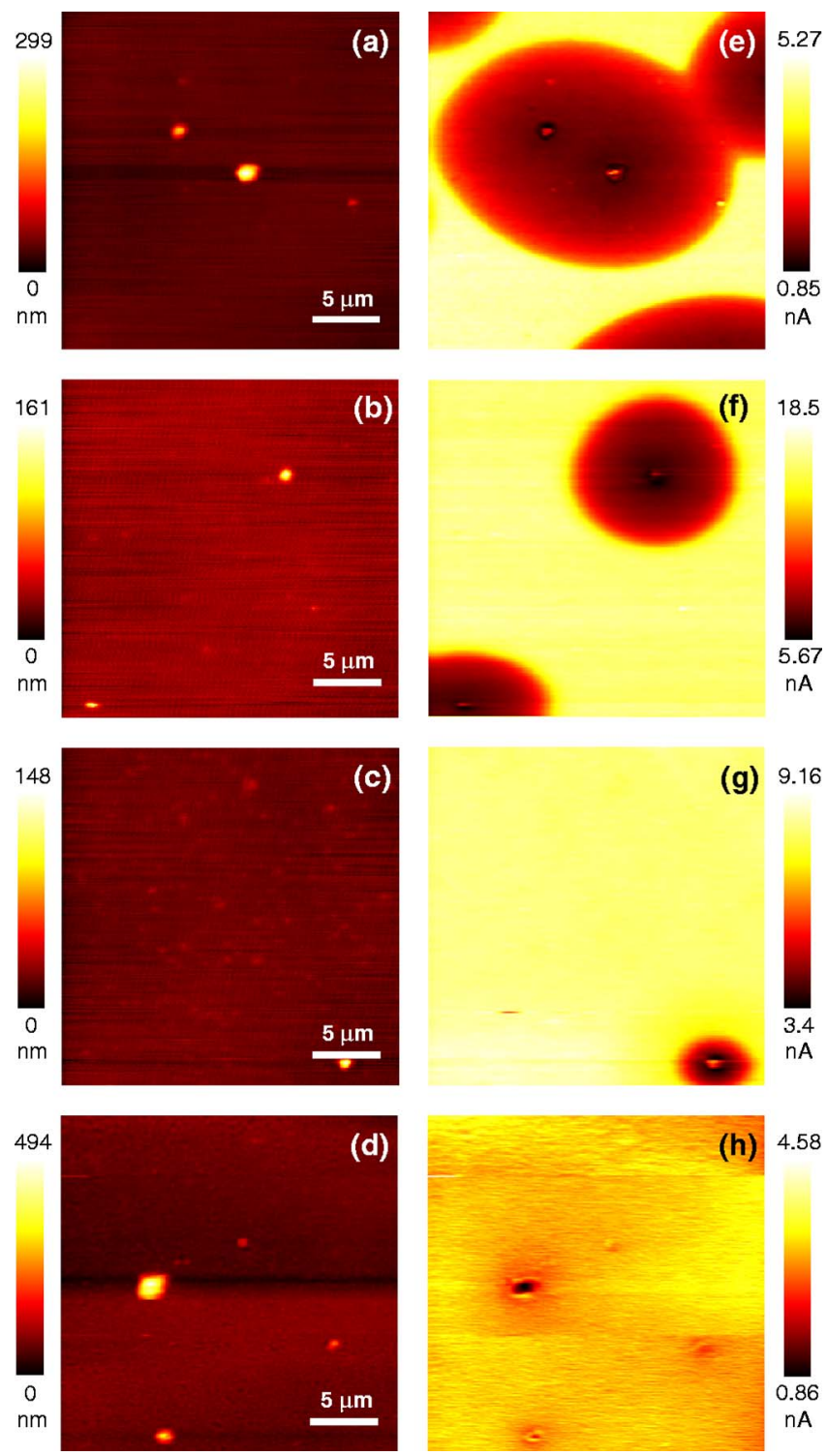

FIG. 3. (Color online) AFM [panels (a)-(d)] and simultaneously collected NSPM [panels (e)-(h)] images of MEH-PPV/PCBM devices. Panels (a) and (e) are for a "cold-spun device" prepared from a chlorobenzene solution. Panels (b) and (f) are for a "room-temperature-spun device" prepared from a chlorobenzene solution. Panels (c) and (g) are for a "hot-spun device" prepared from a chlorobenzene solution. Panels (d) and (h) for a device prepared from a toluene solution. A tip aperture of $200 \mathrm{~nm}$ was used to obtain these images. tered on vertical "spikes" in the corresponding AFM images with little indication from the height image as to the lateral extent of the current depletion region. The fact that the spikes are also observed in regions of the film which are uncoated with metal indicates that they are not due to defects or structures in the top electrode.

In addition, there is a systematic increase in the size and frequency of the micron-sized current-depleted regions with increasing solvent evaporation time, with the cold-spun film allowed the longest time to dry and the hot-spun device the least. Although the toluene devices are outperformed by chlorobenzene devices, ${ }^{4}$ somewhat surprisingly no largescale current depletion regions are observed in the tolueneprepared device.

In order to provide a more quantitative measure of the pattern formation process, three characteristic parameters have been extracted from the images shown in Fig. 3. First, the average diameter of the current depletion regions in the NSPM maps has been measured. Second, by determining an appropriate threshold photocurrent level, each pixel in the NSPM image corresponding to a current depletion region can be identified and hence the proportion of the total device surface area that consists of current-depleted regions calculated. Third, by characterizing a spike as a feature in the AFM images whose height from the surface is $30 \mathrm{~nm}$ or higher, the size and frequency of the spikes were measured. The results of these analyses were averaged over the five scans in total taken for each device and are presented in Table I.

The results shown in Table I confirm that there is a systematic increase in the average size and extent of the current depletion regions with increasing solvent evaporation time. In particular, while only $4 \%$ of the hot-spun device area is depleted with an average spot size of $8 \mu \mathrm{m}, 17 \%$ of the device area of the room-temperature-spun device is depleted with an average spot diameter of $13 \mu \mathrm{m}$, while $50 \%$ of the cold-spun device area is depleted with an average depletion diameter of $20 \mu \mathrm{m}$. This systematic increase in spot size and coverage with solvent evaporation time suggests that these features are built up during film formation. Similarly, the average spike height is also observed to systematically increase with increasing solvent evaporation time consistent with their formation during spin coating. Interestingly, the number of spikes in the film does not change systematically

TABLE I. Characteristics of the NSFM and AFM images for MEH-PPV/PCBM blend films prepared under different processing conditions and measured near- and far-field currents.

\begin{tabular}{|c|c|c|c|c|c|c|}
\hline Device & $\begin{array}{l}\text { Average } \\
\text { diameter } \\
\text { of current- } \\
\text { depleted } \\
\text { region } \\
(\mu \mathrm{m})\end{array}$ & $\begin{array}{c}\text { Area } \\
\text { current- } \\
\text { depleted } \\
(\%)\end{array}$ & $\begin{array}{c}\text { Average } \\
\text { spike } \\
\text { height } \\
(\mathrm{nm})\end{array}$ & $\begin{array}{c}\text { Total } \\
\text { number } \\
\text { of spikes } \\
\text { in the } 5 \\
\text { scans }\end{array}$ & $\begin{array}{c}\text { Integrated } \\
\text { near-field } \\
I_{\mathrm{SC}} \\
\text { (a.u.) }\end{array}$ & $\begin{array}{c}\text { Far-field } \\
I_{\mathrm{SC}} \\
\left(\mathrm{mA} / \mathrm{cm}^{2}\right)\end{array}$ \\
\hline Cold-spun & 20.5 & 50 & 100 & 17 & 1.19 & 1.9 \\
\hline $\begin{array}{l}\text { Room- } \\
\text { temperature-spun }\end{array}$ & 13.1 & 17 & 96 & 9 & 4.52 & 2.2 \\
\hline Hot-spun & 8.1 & 4 & 74 & 12 & 2.95 & 1.9 \\
\hline Toluene & $\left(<0.5^{\mathrm{a}}\right)$ & $\cdots$ & 117 & 47 & 0.94 & 0.9 \\
\hline
\end{tabular}

${ }^{\mathrm{a}}$ Reference 13 . 

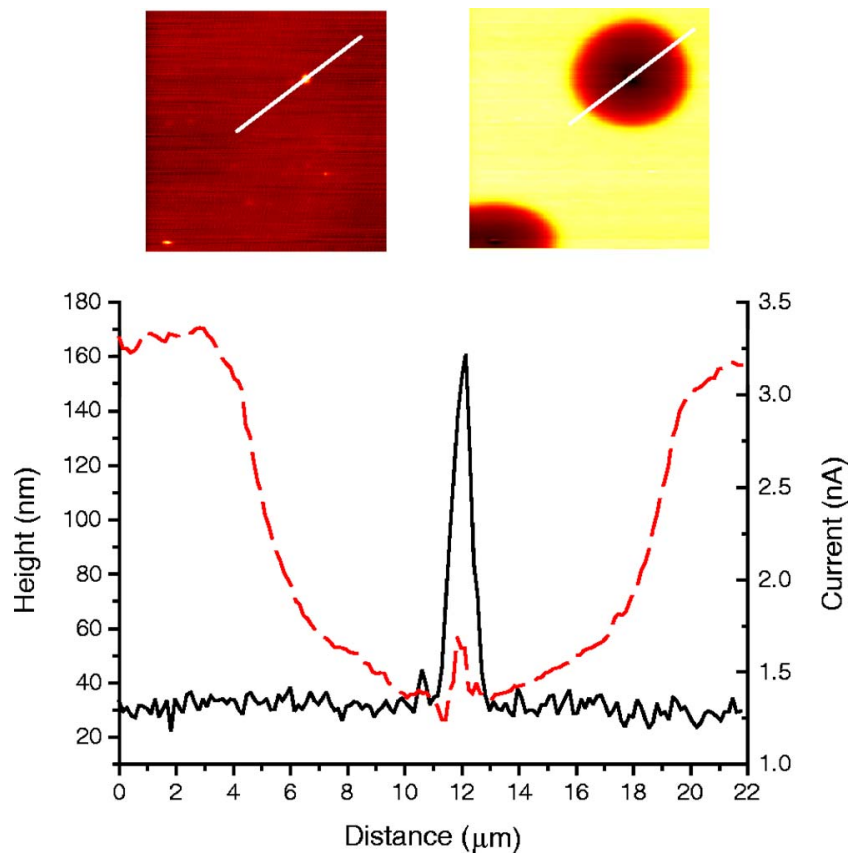

FIG. 4. (Color online) Cross-sectional height (solid) and current (dashed) traces of the room-temperature-prepared device.

with film drying time and may indicate that the initial nucleation density is independent of the processing conditions. However, given relatively low statistics presented in Table I, further experiments are required to fully elucidate this point.

Therefore, it would seem that both spike size and current depletion area increase with increasing solvent evaporation time. In order to further quantify this relationship, the volume of individual current-depleted "black spots" has been plotted against the volume of their central spike. On the basis of the cross-sectional data shown in Fig. 4, the geometry of the current depletion and central spike regions can be simply modeled as cylinders and cones, respectively. As shown in Fig. 5, there is indeed a strong correlation between the vol-

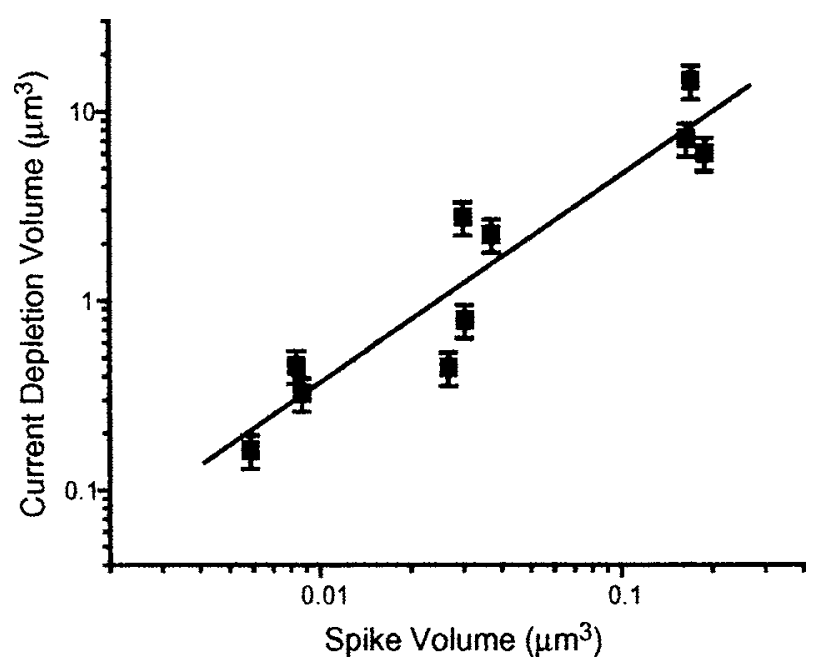

FIG. 5. Plot of current depletion volume vs spike volume demonstrating that the spike is built up from PCBM sourced from the surrounding region. The error bars are an estimate of the error associated with fitting a cylinder with sharp edges to the depletion regions that have a less defined boundary as illustrated in Fig. 4. ume of the central spike and the volume of the surrounding current depletion region, which indicates that the central spike is built up from material sourced from the surrounding current depletion region.

The observation of a quantitative relationship between spike size, current depletion size, and solvent evaporation time are consistent with the segregation of the two components in the PCBM/MEH-PPV polymer blend, which is known to occur in these materials ${ }^{7,8,10}$ but not previously observed on a micrometer length scale. It is worth noting, however, that micron-sized phase segregation of PCBM in blend films of PCBM/poly(3-hexylthiophene) (PCBM/ $\mathrm{P} 3 \mathrm{HT}$ ) with high loadings of P3HT has been observed recently using AFM. ${ }^{9}$ The observation of a central spike surrounded by a poor photocurrent-producing region suggests that PCBM clusters nucleate and grow during film formation, with the reduction in photocurrent collection efficiency arising from the depletion of PCBM from the surrounding regions. The observation that no micron-sized current features are observed in the toluene-prepared device, despite showing large variation in current on the submicron scale as previously imaged by NSPM, ${ }^{13}$ is consistent with the lower solubility of PCBM in toluene, which prevents large-scale migration during film formation. The faster evaporation rate of toluene compared to chlorobenzene is also expected to be a limiting factor, but the presence of large numbers of PCBM aggregates in the toluene device (which was prepared in otherwise identical fashion to the chlorobenzene devices) indicates that PCBM solubility is actually the key factor.

Varying the evaporation time necessarily alters other important experimental parameters, namely, solution temperature and concentration, and thus we need to consider the influence of these parameters upon the proposed mechanism. As solution temperature is varied, it can be expected that the mobility of PCBM in solution also varies, with higher mobilities at higher temperatures. However, since the greatest extent of PCBM segregation was observed with the coldspun film, these observations cannot be attributed to variations in PCBM mobility. Solution-concentration-induced changes in solubility cannot explain the effects seen either, since the hot-spun device showed lower numbers of aggregates than the cold-spun device despite being processed from a solution with higher concentration.

Low solution temperatures could lead to a reduction in PCBM solubility, which could account for larger number of aggregates observed in the cold-spun device, as is observed for toluene-spun films due to the lower solubility of PCBM in toluene compared to chlorobenzene. However, lower PCBM solubility should also be accompanied by a reduction in the size of the current-depleted regions, which is not observed in Fig. 3. We can also discount the possibility that the differences in the number of aggregates arise from variations in the number nucleation sites due to differences in impurity density on the substrate, as all devices were prepared identically. Therefore, the nucleation and growth of PCBM aggregates during film formation is the most likely self-consistent explanation for the features observed.

The dramatic reduction in photocurrent efficiency of the regions surrounding the PCBM aggregates can also be ex- 


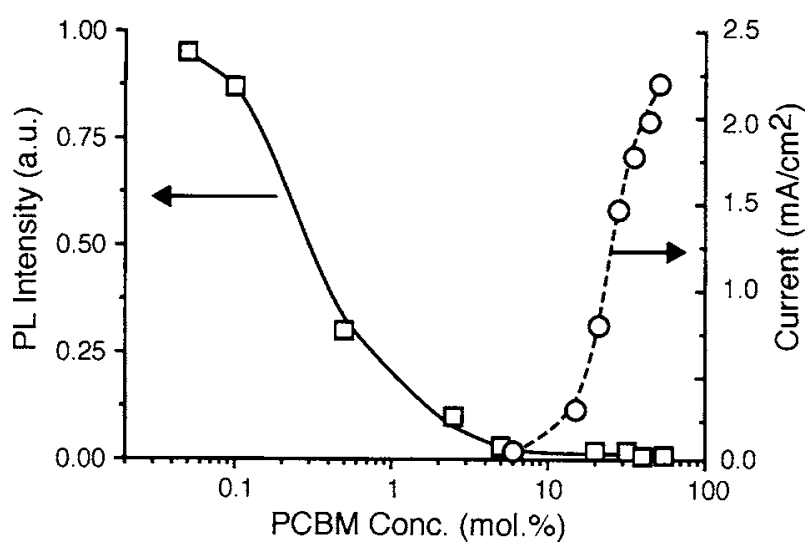

FIG. 6. Variation of short-circuit current (circles, dashed line) for chlorobenzene-spun MEH-PPV/PCBM blend films as a function of PCBM concentration in mol \% on logarithmic scale with the variation of photoluminescence (squares, solid line) as a function of PCBM concentration plotted for comparison. All devices were prepared at room temperature, with short-circuit current measured under $\sim 80 \mathrm{~mW} / \mathrm{cm}^{2}$ white light. The lines are guides to the eye.

plained in terms of a segregation mechanism. As shown in Fig. 6, there is a significant difference in the dependence of the photocurrent signal on PCBM concentration compared to the variation in photoluminescence, in agreement with results reported elsewhere. ${ }^{7,14,15}$ In particular, the data of Fig. 6 show that while fluorescence measurements are most sensitive to variations in PCBM concentration between 0.5 and 5 wt $\%$ (or 0.1 and $1 \mathrm{~mol} \%$ ), the current mapping measurements have greatest sensitivity to PCBM concentration variations between 40 and $80 \mathrm{wt} \%$ (or 20 and $50 \mathrm{~mol} \%$ ). Since the degree of photoluminescence quenching is representative of the efficiency of charge generation, Fig. 6 highlights the two separate conditions that need to be achieved for efficient polymeric photovoltaic devices, namely, efficient charge generation and charge collection. ${ }^{16}$ Thus while other techniques, such as fluorescence NSOM, are often employed for probing local device functionality, ${ }^{17,18}$ NSPM is unique in that it allows a direct measurement of local charge generation and collection efficiency with subwavelength resolution. The sharp rise in current at around 50 wt \% PCBM can be attributed to the establishment of efficient charge transport networks coinciding with the onset of PCBM phase segregation. ${ }^{7}$ Thus large variations in current as observed by NSPM are brought about by relatively small changes in PCBM concentration of between 50 and $80 \mathrm{wt} \%$.

The cross-sectional trace of Fig. 4 for the roomtemperature-spun device shows that the magnitude of the photocurrent at the base of the current depletion region $(\sim 7 \mathrm{nA})$ is approximately $45 \%$ of its value $(\sim 16.5 \mathrm{nA})$ in the surrounding nondepleted area, similar to observations by Jeranko et al. ${ }^{11}$ The photocurrent is also observed to drop by a similar proportion in the current-depleted regions of the cold-spun and hot-spun devices. Assuming that the nondepleted regions maintain a composition of $80 \mathrm{wt} \%$ PCBM, reading off Fig. 6 the PCBM concentration where current drops to $45 \%$ of its peak value provides an estimated PCBM concentration in the depleted regions of $29 \mathrm{~mol} \%$ or 55

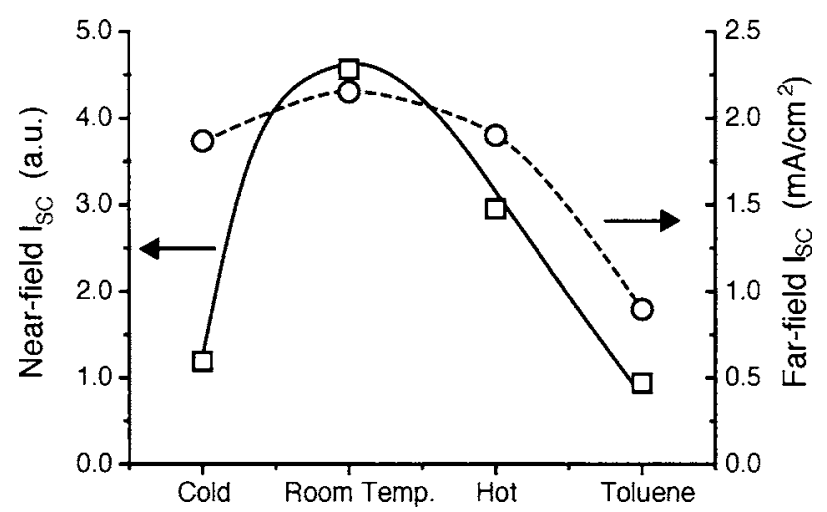

FIG. 7. Comparison of near-field (squares) and far-field (circles) measured short-circuit currents for the variously prepared devices.

wt $\%$. Thus the current depletion regions are not entirely depleted of PCBM and still contain a significant proportion of methanofullerene.

It is clear, therefore, that the contrast observed in the NSPM images can be explained by an actual change in PCBM concentration from 80 to $55 \mathrm{wt} \%$. Moreover, at the boundary of the current-depleted region there is no change in physical height of the film. As such, the contrast mechanisms that operate in more conventional imaging techniques, such as TEM, AFM, and fluorescence spectroscopy, are not sensitive enough to detect these subtle changes, providing an explanation as to why these micron-sized current depletion regions have not been previously reported by these techniques. These relatively small changes in PCBM concentration nevertheless have a dramatic effect on photocurrent generation, and demonstrates that NSPM is able to detect subtle changes in film composition as they affect device performance.

Figure 7 shows the far-field short-circuit currents (measured under $\sim 80 \mathrm{~mW} / \mathrm{cm}^{2}$ white light) and integrated nearfield currents for the four different devices. It should be noted that all devices showed similar open-circuit voltages $(\sim 0.75 \mathrm{~V})$ and fill factors $(\sim 0.45)$ under white-light illumination and therefore the far-field short-circuit currents can be taken as a measure of overall efficiency. As the same NSOM tip was used throughout the NSPM study, a similar flux of light was delivered for all the near-field measurements, allowing for a quantitative comparison of the near-field photocurrents. The integrated near-field current values were calculated by summing the absolute current values recorded over each of the five scans taken per device type. The same general current trend over the four device types is observed for both near-field and far-field measurements, with the toluene device measuring the lowest far-field short-circuit current, followed by the cold-spun and hot-spun devices. We can discount the possibility that these short-circuit current variations are attributed to any variation in active layer thickness since power conversion efficiency is observed to vary by less than $10 \%$ as film thickness varies from 50 to $500 \mathrm{~nm},{ }^{5}$ and external quantum efficiency (EQE) by a similar amount as thickness varies from 70 to $350 \mathrm{~nm} .{ }^{19}$ The short-circuit data presented in Fig. 7 are also summarized in Table I. Note that while the short-circuit currents presented here are lower than those reported elsewhere, ${ }^{4}$ they are comparable with those 
reported recently by van Duren $e t a l .^{7}$ and the use of a nonstandard white-light source used here makes an absolute comparison difficult. However, given that we see the same efficiency trends between the chlorobenzene and tolueneprepared devices and we have observed the same nanomorphology relationships ${ }^{13}$ as elsewhere ${ }^{4,8,10}$ indicates that the effects presented here are general.

Given the systematic decrease in the size and frequency of the current depletion regions with decreasing evaporation time, it might be expected that the hot-spun device would outperform both the room-temperature- and cold-spun devices. However, as illustrated in Fig. 7, this trend was not observed and, in fact, the short-circuit current performance is optimized for the room-temperature-spun device, even though a higher proportion of its device area consists of current-depleted regions. As such, it would appear that the variation in device performance is not governed primarily by the observed micron-scaled features. We speculate that the variation in spin conditions also produces varying amounts of nanoscale phase separation, which ultimately dictates device performance. Such an explanation is consistent with the findings of van Duren $e t \mathrm{al}^{7}$ who concluded that a minimum PCBM domain size is necessary for efficient charge transport, and the results of Yang et al. ${ }^{20}$ and Shaheen et $_{\text {al. }}{ }^{21}$ who observed larger phase-segregated domain sizes in $80 \mathrm{wt} \%$ PCBM composite films formed with longer solvent evaporation times. For the devices prepared here, the roomtemperature-spun device therefore provides the optimum balance between nanoscale phase separation and micron-sized current depletion. While the hot-spun device exhibited the least amount of micron-sized current depletion, it is argued that the spin speed and temperature of the solution did not allow enough time for the formation of sufficient nanoscale phase separation necessary for optimal charge transport. Indeed, examining the NSPM current images (Fig. 3) reveals that the photocurrent in the nondepleted regions of the roomtemperature-spun device is twice that of the current produced by the hot-spun device. Thus it is the efficiency of charge collection in the nondepleted regions that ultimately determines the overall performance of these devices. The fact that no variation in photocurrent in the nondepleted regions for the cold-, room-temperature-, and hot-spun devices is observed suggests that the length scale of this nanoscale phase separation is significantly less than the $200 \mathrm{~nm}$ tip aperture dimension used in the experiments presented here. Indeed, the high-resolution (100 $\mathrm{nm}$ aperture) image presented in Fig. 8 for a nondepleted region in the room-temperature device shows that photocurrent varies by less than $2 \%$ over the scan area $1.2 \times 1.2 \mu \mathrm{m}^{2}$. Although the image shown in Fig. 8 shows that there is some correlation between the nanoscale morphology and photocurrent (marked regions in scan), the present resolution of the NSPM technique is still insufficient to resolve the finer scale features that determine overall device performance.

Another feature presented in the comparison of nearfield and far-field currents in Fig. 7 is that while both far- and near-field current measurements follow the same trend for the four devices tested, the magnitude of the variation in overall photocurrent is much greater for the near-field than
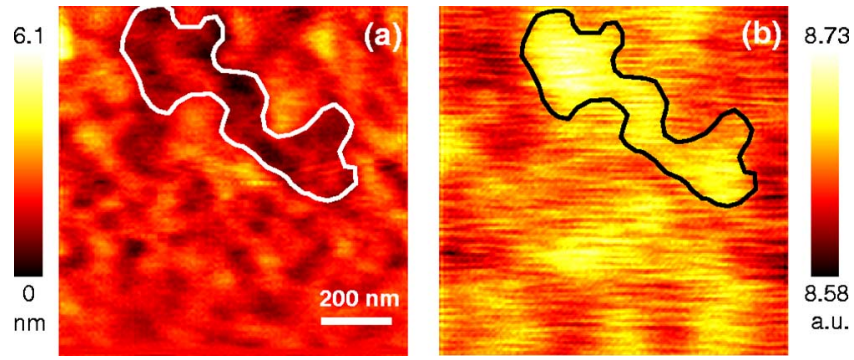

FIG. 8. (Color online) AFM (a) and simultaneously collected NSPM (b) images of a nondepleted region in the room-temperature-spun device. There is a less than $2 \%$ variation in current across the scan area. The outlined regions highlight the correlation between morphology and current in this device on the nanoscale. A tip aperture of $100 \mathrm{~nm}$ was used.

for the far-field measurements. In particular, the near-field measurement of the integrated current of the cold-spun device is almost $74 \%$ less than that of the room-temperaturespun device. Moreover, for the three chlorobenzene-prepared devices the measured far-field short-circuit current varies by less than $14 \%$ despite the large variation in both the extent of the current depletion regions and the magnitude of current observed in the nondepleted regions.

The difference between the near-field and far-field measurements can be explained by the different illumination directions for the two measurements. For the far-field measurements, light is injected through the ITO/PEDOT:PSS anode, while for the near-field measurements light was injected through the metal cathode. These different illumination directions necessarily lead to different exciton density gradients across the cell. Thus, the maximum exciton density occurs close to the anode for the far-field measurements and close to the cathode for the near-field measurements. It is well established that a concentration of $30 \mathrm{wt} \%$ PCBM is sufficient to produce a charge generation efficiency of virtually unity. ${ }^{7}$ From the preceding discussion, it was estimated that the minimum PCBM concentration in the film that occurs in the current-depleted regions is approximately 55 wt \%. As such, there is sufficient PCBM throughout the film to ensure that charge generation is uniform throughout the film, even in the current depletion regions, and so the differences in far-field and near-field performances observed in Fig. 7 must arise from differences in charge collection efficiency. The observation that the greatest variation in overall current occurs when the photoexcitations are localized near the cathode implies that the charge collection efficiency is dominated by hole, rather than electron, transport effects. Such an observation seems contrary to expectation given that it is generally accepted that high loadings of PCBM are required for efficient electron transport in the composite, with hole mobility, as afforded by the polymer, remaining relatively insensitive to morphology and PCBM concentration. ${ }^{22}$ However, recent reports by Mihailetchi et al. ${ }^{23}$ and Tuladhar et $\mathrm{al}^{24}$ show that the hole transport in MDMO-PPV/PCBM composites indeed depends on PCBM concentration. These reports demonstrate that similar to electron mobility, hole mobility increases by over three orders of magnitude as the PCBM concentration is increased from 15 to $70 \mathrm{wt} \%{ }^{23,24}$ Furthermore, since the hole mobility is actually lower than 
the electron mobility in MDMO-PPV/PCBM composites for a range of PCBM concentrations, it appears that hole transport is actually more sensitive than electron transport to subtle variations in PCBM concentration. In addition, the much higher light intensities used in the near-field measurements (estimated at $50 \mathrm{~kW} / \mathrm{cm}^{2}$ assuming a tip transmission of $0.1 \%$ and cathode transparency of $20 \%$ for an input power of $10 \mathrm{~mW} / \mathrm{cm}^{2}$ ) produce higher charge-carrier densities in the material, which would tend to accentuate any PCBMdependent variations in hole mobility due to space-charge effects. ${ }^{25}$ Nonlinear effects may also be important, and further studies into the influence of high laser powers on device performance would allow for better comparability of nearfield and far-field measurements. Nevertheless, the results presented here, in addition to the work of Mihailetchi et al..$^{23}$ and Tuladhar et al. ${ }^{24}$ perhaps highlight the need to reassess whether the strong dependence of efficiency on PCBM concentration and morphology is a product of electron transport effects alone, or whether hole mobility also plays a significant role.

\section{CONCLUSIONS}

By observing the dependence of processing conditions on the size and frequency of the micron-sized current depletion regions observed in NSPM images of chlorobenzeneprocessed PPV/PCBM solar cells we have shown that they are the result of the nucleation and growth of the central PCBM cluster at the expense of PCBM from the surrounding area. By varying the time allowed for the film to dry, the diameter of these regions was observed to vary between 5 and $25 \mu \mathrm{m}$, with the proportion of the total device area covered by these regions varying between $4 \%$ and $50 \%$. The correlation between the volume of the central spike and the surrounding depletion volume indicates that the central spike is built up during film formation from material sourced from the surrounding region. Comparing the magnitude of current depletion to far-field measurements of the dependence of short-circuit current on PCBM concentration, the PCBM concentration in these regions was estimated to be $55 \mathrm{wt} \%$, indicating that they still contain significant amounts of PCBM. The absence of large-scale current depletion regions in toluene-prepared devices provides further evidence that the formation of these features is related to the mobility of PCBM in the film during drying, since PCBM has twice the solubility in chlorobenzene than in toluene. Comparison of the far-field device performance indicated that chlorobenzene devices consistently outperformed the toluene devices, despite the varying degrees of current depletion in the chlorobenzene devices (4\% of total device area to $50 \%$ of total area, varied by varying the film drying time). Surprisingly, device performance was maximized for devices spin coated at room temperature at a moderate spin speed, rather than for conditions that minimized the extent of the current depletion regions. This trend was interpreted in terms of the influence of the nano scale morphology, with optimum device performance resulting from a balance between the effects of nanoand micron-scale segregations. The fact that micron-sized current depletion regions are still observed in such an optimized device suggests that further improvements in efficiency can be gained.

\section{ACKNOWLEDGMENTS}

This work was carried out with the support of the Australian Research Council. One of the authors (C.R.M.) is grateful to the University of Newcastle for financial support.

${ }^{1}$ G. Yu, J. Gao, J. C. Hummelen, F. Wudl, and A. J. Heeger, Science 270, 1789 (1995)

${ }^{2}$ C. J. Brabec, F. Padinger, J. C. Hummelen, R. A. J. Janssen, and N. S. Sariciftci, Synth. Met. 102, 861 (1999).

${ }^{3}$ C. J. Brabec, N. S. Sariciftci, and J. C. Hummelen, Adv. Funct. Mater. 11, 15 (2001).

${ }^{4}$ S. E. Shaheen, C. J. Brabec, N. S. Sariciftci, F. Padinger, T. Fromherz, and J. C. Hummelen, Appl. Phys. Lett. 78, 841 (2001).

${ }^{5}$ T. Munters et al., Thin Solid Films 403-404, 247 (2002).

${ }^{6}$ F. Padinger, R. S. Rittberger, and N. S. Sariciftci, Adv. Funct. Mater. 13, 85 (2003).

${ }^{7}$ J. K. L. van Duren, X. Yang, J. Loos, C. W. T. Bullie-Lieuwma, A. B. Sieval, J. C. Hummelen, and R. A. J. Janssen, Adv. Funct. Mater. 14, 425 (2004).

${ }^{8}$ H. Hoppe, M. Niggemann, C. Winder, J. Kraut, R. Hiesgen, A. Hinsch, D. Meissner, and N. S. Sariciftci, Adv. Funct. Mater. 14, 1005 (2004).

${ }^{9}$ D. Chirvase, J. Parisi, J. C. Hummelen, and V. Dyakonov, Nanotechnology 15, 1317 (2004)

${ }^{10}$ T. Martens et al., Synth. Met. 138, 243 (2003).

${ }^{11}$ T. Jaernko, H. Tributscha, N. S. Sariciftci, and J. C. Hummelen, Sol. Energy Mater. Sol. Cells 83, 247 (2004).

${ }^{12}$ C. R. McNeill, H. Frohne, J. L. Holdsworth, J. E. Furst, B. V. King, and P. C. Dastoor, Nano Lett. 4, 219 (2004).

${ }^{13}$ C. R. McNeill, H. Frohne, J. L. Holdsworth, and P. C. Dastoor, Synth. Met. 147, 107 (2004).

${ }^{14}$ A. Haugeneder et al., Phys. Rev. B 59, 15346 (1999).

${ }^{15}$ S. Morita, S. Kiyomatsu, X. H. Yin, A. A. Zakhidov, T. Noguchi, T. Ohnishi, and K. Yoshino, J. Appl. Phys. 74, 2860 (1993).

${ }^{16}$ C. R. McNeill and P. C. Dastoor, in Electronic and Optical Properties of Conjugated Molecular Systems in Condensed Phases, edited by S. Hotta (Research Signpost, Trivandrum, 2003), pp. 377-418.

${ }^{17}$ A. C. Arias, J. D. MacKenzie, R. Stevenson, J. J. M. Halls, M. Inbasekaran, E. P. Woo, D. Richards, and R. H. Friend, Macromolecules 34, 6005 (2001).

${ }^{18}$ T. Röder, H.-S. Kitzerow, and J. C. Hummelen, Synth. Met. 141, 271 (2004).

${ }^{19}$ R. Schilinksky, C. Waldauf, and C. J. Brabec, Appl. Phys. Lett. 81, 3885 (2002).

${ }^{20}$ X. Yang, J. K. L. van Duren, R. A. J. Janssen, M. A. J. Michels, and J. Loos, Macromolecules 37, 2151 (2004).

${ }^{21}$ S. E. Shaheen, R. Radspinner, N. Peyghambarian, and G. E. Jabbour, Appl. Phys. Lett. 79, 2996 (2001).

${ }^{22}$ H. Hoppe and N. S. Sariciftci, J. Mater. Res. 19, 1924 (2004).

${ }^{23}$ V. D. Mihailetchi, L. J. A. Koster, P. W. M. Blom, C. Melzer, B. de Boer, J. K. L. van Duren, and R. A. J. Janssen, Adv. Funct. Mater. 15, 795 (2005).

${ }^{24}$ S. M. Tuladhar, D. Poplavskyy, S. A. Choulis, J. R. Durrant, D. C. Bradley, and J. Nelson, Adv. Funct. Mater. 15, 1171 (2005).

${ }^{25}$ V. D. Mihailetchi, J. Wildeman, and P. W. M. Blom, Phys. Rev. Lett. 94, $126602(2005)$. 